Supporting Information

\title{
Ultrafast Triplet Generation at the Lead Halide Perovskite/Rubrene
}

\section{Interface}

Carl R. Conti III, ${ }^{1, \#}$ Alexander S. Bieber, ${ }^{1, \#}$ Zachary A. VanOrman, ${ }^{1}$ Gregory Moller, ${ }^{1}$ Sarah

Wieghold, ${ }^{1,2,3}$ Richard D. Schaller, ${ }^{2}$ Geoffrey F. Strouse, ${ }^{1}$ Lea Nienhaus ${ }^{1, *}$

${ }^{1}$ Department of Chemistry and Biochemistry, Florida State University, Tallahassee, FL 32306, USA

${ }^{2}$ Center for Nanoscale Materials, Argonne National Laboratory, Lemont, IL 60439, USA

${ }^{3}$ Advanced Photon Source, Argonne National Laboratory, Lemont, IL 60439, USA

\#equal contribution

*corresponding Author: lnienhaus@fsu.edu 


\section{Experimental Methods}

\section{Materials}

$\mathrm{PbI}_{2}$ (99.99\%, Tokyo Chemical Industries), MAI (Dyenamo), FAI (Dyenamo), dibenzotetraphenylperiflanthene (DBP, 99\%, Sigma Aldrich), rubrene (99.99\%, Sigma Aldrich), toluene (anhydrous, Sigma Aldrich), chlorobenzene (anhydrous, Sigma Aldrich), dimethylformamide (DMF, anhydrous, Sigma Aldrich), dimethyl sulfoxide (DMSO, anhydrous, Sigma Aldrich). All chemicals were used as received.

\section{Device Fabrication}

Perovskite thin films and UC devices were fabricated using methods similar to previously described ones. ${ }^{1,2}$ Glass substrates were cleaned via subsequent sonication for $15 \mathrm{~min}$ in $2 \%$ Hellmanex solution, deionized water and ethanol, respectively. Substrates were then wiped with acetone and treated by UV-Ozone plasma (Ossila) for $15 \mathrm{~min}$. A perovskite precursor solution was made by combining 1.2 $\mathrm{M}$ precursor solutions of MAI, FAI, and $\mathrm{PbI}_{2}$, all in 9:1 DMF:DMSO (v:v). The precursor solution was diluted 5-fold to create sufficiently thin films (thickness estimated at $\sim 30 \mathrm{~nm}$ ) to enable TA measurements. The precursor solution was added to the glass substrate prior to a two-step spin-coating program, $1000 \mathrm{rpm}$ for $10 \mathrm{~s}$ and $5000 \mathrm{rpm}$ for $30 \mathrm{~s}$. Chlorobenzene was used as the antisolvent, and the resulting thin films were annealed at $120{ }^{\circ} \mathrm{C}$ for $10 \mathrm{~min}$ in an air-free environment.

For bilayer devices, rubrene $(10 \mathrm{mg} / \mathrm{mL})$ doped with $1 \mathrm{wt} \%$ DBP in toluene, was deposited onto the perovskite film and spin coated at $6000 \mathrm{rpm}$ for $20 \mathrm{~s}$. All perovskite thin films and bilayer devices were sealed with a coverslip and a two-component epoxy resin (Devcon) under an inert nitrogen atmosphere. 


\section{Transient Absorption Spectroscopy}

Transient absorption measurements were performed using a HELIOS Fire transient absorption spectrometer (Ultrafast Systems). Femtosecond pulses were generated using a Coherent Astrella-V-F-1K amplifier system. A Vitara-S Coherent Ti:Sapphire laser was amplified using a 1 $\mathrm{kHz}$ Coherent Revolution-50 pump laser and generated a $5 \mathrm{~mJ}$ pulse with a 100 fs full width half $\max$ at $800 \mathrm{~nm}$. The pulse was split into a pump and a probe beam, where the probe was directed through a delay stage, while the pump passed through an optical parametric amplifier (OPerA Solo, Coherent). The white light continuum used for the probe was produced by a $\mathrm{CaF}_{2}$ crystal for all measurements. A dual chopper setup was implemented to eliminate pump scatter. The pump and probe beam were then overlapped on the thin film sample. Difference spectra and single wavelength kinetics were collected averaging 3 times and holding for 2 seconds, with an exponential point acquisition beginning with $0.01 \mathrm{ps}$ steps and totaling to 150 points (Figure 1). Pump powers were attenuated with neutral density filters.

The power-dependent transient absorption measurements (Figure 2 and Figures S3 and S4) and transient absorption measurements of rubrene/DBP (Figure $\mathrm{S} 1$ ) and $\mathrm{PbI}_{2}$ control samples (Figure S7) were performed using a 35 fs, 2kHz Spectra Physics Spitfire amplified Ti:Sapphire laser that produces $2 \mathrm{~mJ}$ per pulse. A portion of this was used to produce white light in a $\mathrm{CaF}_{2}$ crystal, and the remainder pumped a Light Conversion Topas parametric amplifier. Powerdependent TA measurements were performed by recording transient absorption scans at indicated time delays followed by motorized adjustment of the laser fluence and repetitious data acquisition.

Raw data was processed using the Surface Xplorer software package from Ultrafast Systems and Matlab. 


\section{Steady-State Absorption Spectroscopy}

Absorption spectra were measured using a Thermo Scientific Evolution 220 Spectrophotometer.

\section{Analysis Methods}

\section{Extraction of the Rubrene PIA}

The extracted rubrene PIA shown in Figure S1b (bottom) is extracted by normalizing the MAFA and MAFA/rub TA spectra at a delay time of $6 \mathrm{~ns}$ at $533 \mathrm{~nm}$. This wavelength is chosen as rubrene is not expected to have a significant PIA at $533 \mathrm{~nm}$ (Figure S1b, top). Subtraction of the normalized MAFA spectrum from the normalized MAFA/rub spectrum yields the residual rubrene-related PIA shown.

\section{Triplet Rise Kinetics Extraction}

To approximate the rate of triplet generation in Figure 3c, we compare the kinetics on the triplet feature to a nearby region which does not exhibit strong triplet absorption. Specifically, the kinetics at $533 \mathrm{~nm}$ are subtracted from the kinetics at $521 \mathrm{~nm}$ for the rubrene triplet and the kinetics at 575 $\mathrm{nm}$ are subtracted from the kinetics at $553 \mathrm{~nm}$ for the residual polaron signal after normalization at $100 \mathrm{ps}$. To obtain the $\mathrm{T}_{1} \rightarrow \mathrm{T}_{\mathrm{n}}$ transition at $485 \mathrm{~nm}$, the underlying MAFA kinetics at $485 \mathrm{~nm}$ are subtracted after normalization of the kinetics between 80-100 ps (compare Figure S6).

\section{Maxwell-Boltzmann Fitting: Hot Carrier Extraction}

Hot carrier temperature and Fermi levels were determined by fitting the high energy side of the feature at $\sim 1.6 \mathrm{eV}$ using the following equation: 


$$
\frac{\Delta T}{T}(E) \propto \exp \frac{E_{F}-E}{k T_{c}}
$$

where delta $\mathrm{T} / \mathrm{T}$ is the normalized differential transmittance of the sample, $\mathrm{E}$ is the energy of the probe photon, $\mathrm{k}_{\mathrm{b}}$ is the Boltzmann constant, $\mathrm{E}_{\mathrm{F}}$ is the hot carrier Fermi level, and $\mathrm{T}_{\mathrm{c}}$ is the hot carrier temperature. Experimentally measured data was initially smoothed for clarity of fitting. The high energy tail was fit for 16 time points for each sample and pump power. Upper and lower boundary conditions for fitting were set to 0.5 and 0.1 for all samples.

\section{Supplemental Figures}
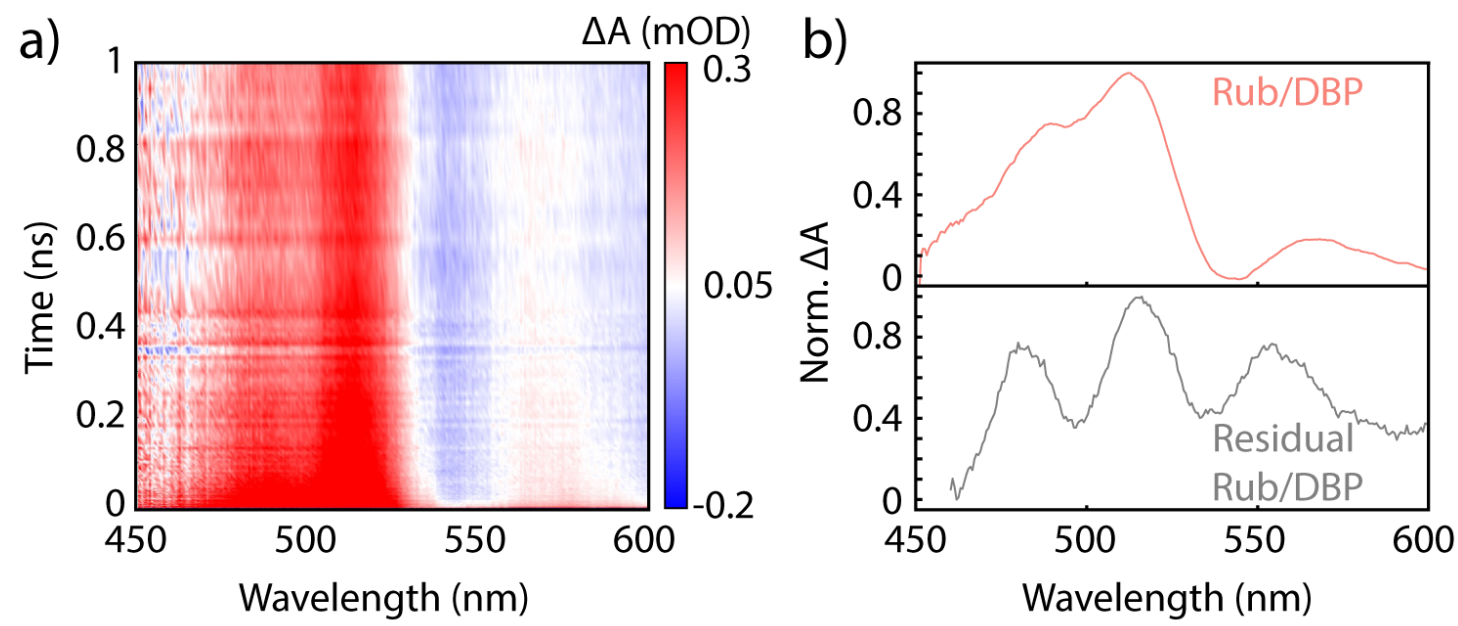

Figure S1: (a) TA surface of a rubrene/DBP film recorded under $420 \mathrm{~nm}$ pump. (b) Normalized TA spectrum of rubrene/DBP (top, pink) at a delay time of $982 \mathrm{~ns}$ recorded under $420 \mathrm{~nm}$ pump, and the extracted rubrene/DBP TA spectrum (gray, bottom) taken by subtracting the MAFA signal from a MAFA/rub spectrum at a delay time of 6 ns recorded under $650 \mathrm{~nm}$ pump. 

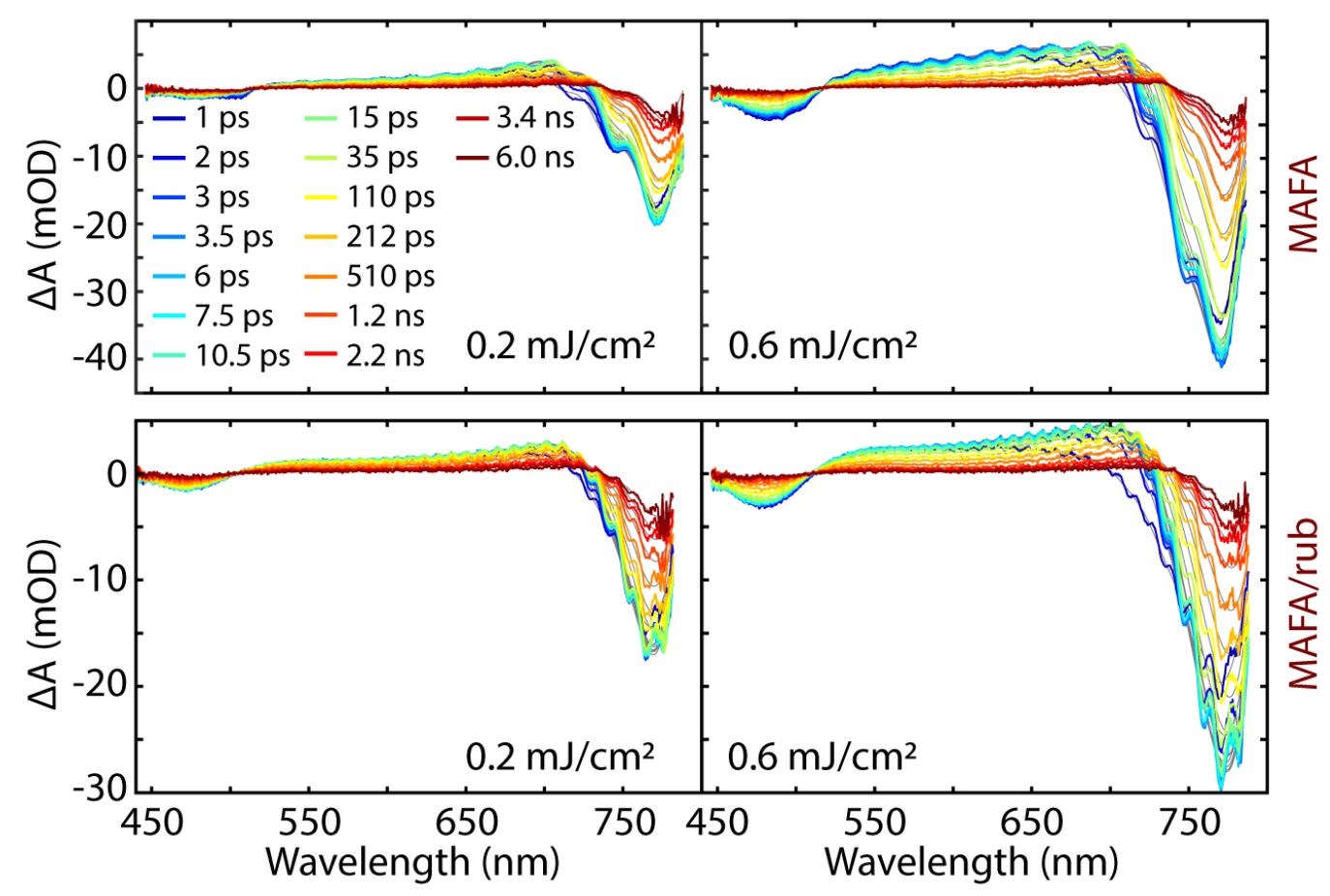

Figure S2: MAFA and MAFA/rub TA spectra at selected delay times recorded under 808 nm pump at power densities of $0.2 \mathrm{~mJ} / \mathrm{cm}^{2}$ (left) and $0.6 \mathrm{~mJ} / \mathrm{cm}^{2}$ (right).

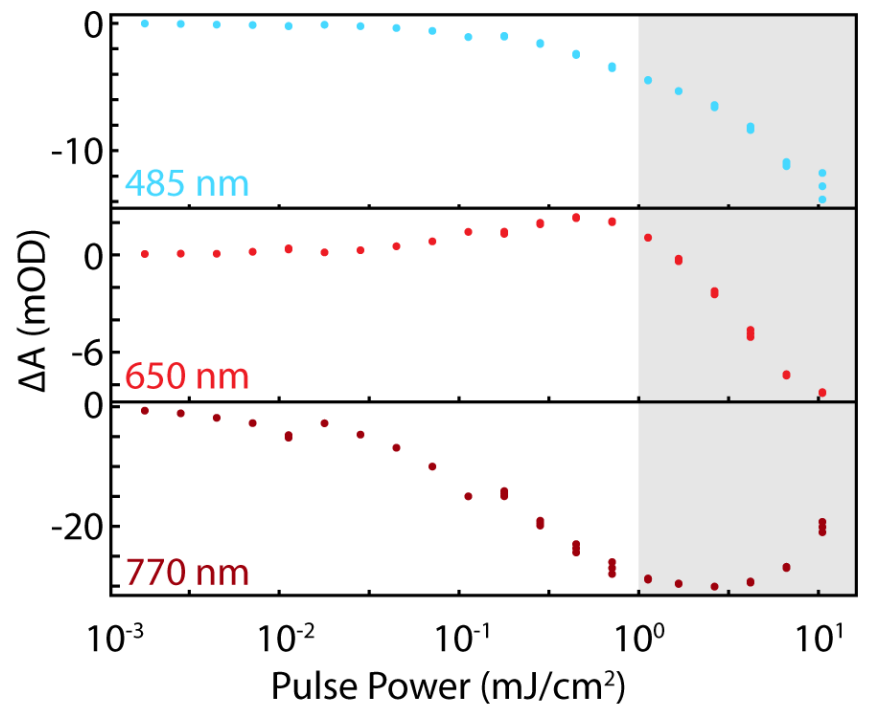

Figure S3: Pulse power dependence of the bleach intensity of PB2 (485 nm), PB3 (650 nm) and PB1 (extracted at 770 $n m$ due to measurement artifact). The shaded region highlights the power regime in which a detectable triplet-related PIA is observed (compare Figure S6). The bleach intensity of PB2 continuously increases with increasing pulse power. The absorbance at $650 \mathrm{~nm}$ is an overlap of PIA1 (positive signal) and PB3, which dominates at high pulse powers, resulting in a negative signal. At high pulse powers PB1 is not yet fully populated at the chosen delay time of 1.5 ps, resulting in the apparent reduction in bleach intensity. 

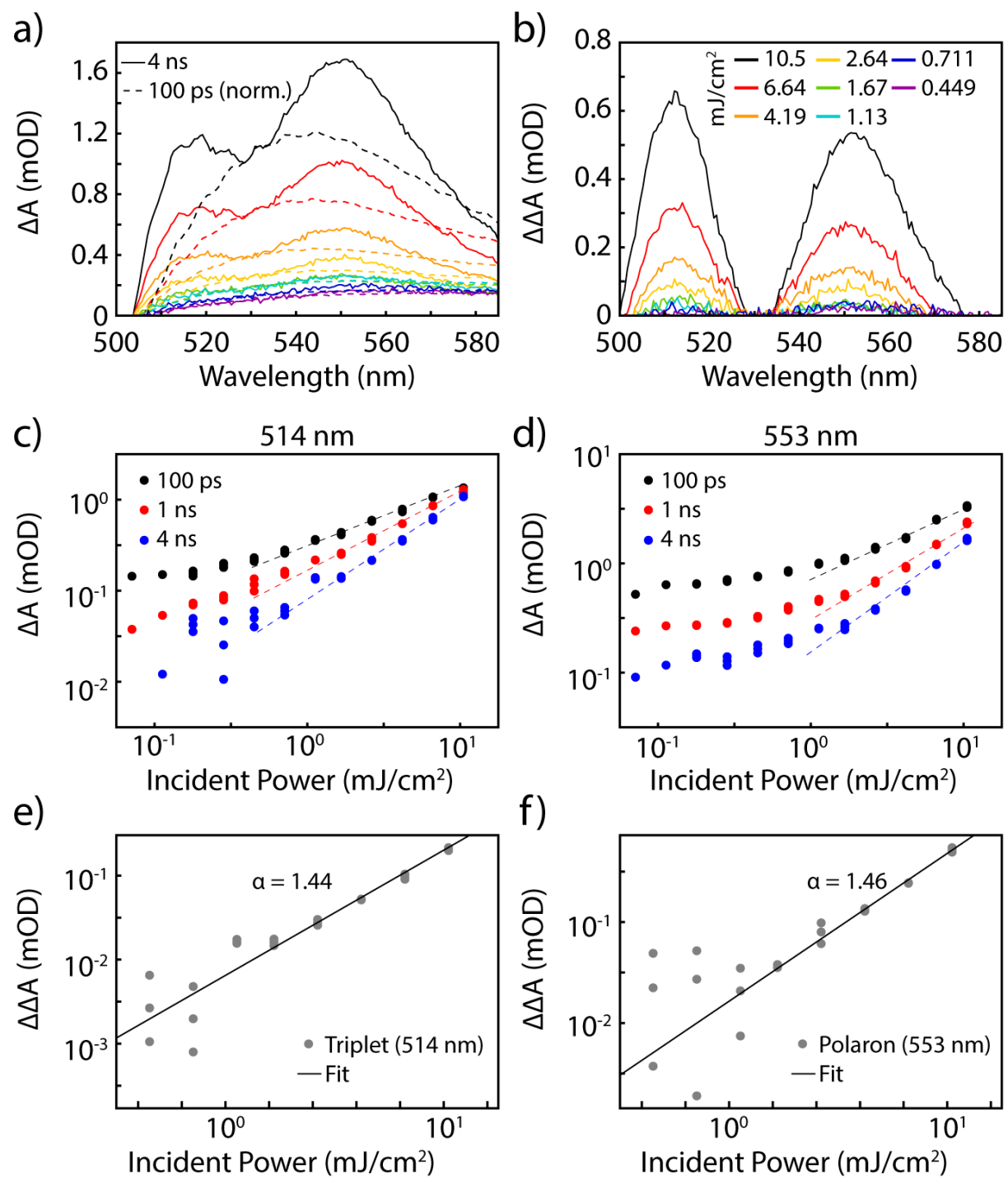

Figure S4: (a) TA spectra of MAFA/rub at selected power densities at a delay time of $4 \mathrm{~ns}$ (solid) and $100 \mathrm{ps}$ (dashed) for three repeated measurements. To obtain the double difference spectra in (b), the data at a delay time of $100 \mathrm{ps}$ was normalized to the value at $533 \mathrm{~nm}$ at a delay time of $4 \mathrm{~ns}$. (b) Double difference TA spectra obtained by subtracting the normalized transient absorbance at $100 \mathrm{ps}$ from the transient absorbance at $4 \mathrm{~ns}$ (compare (a)). (c) and (d) loglog plot of the MAFA/rub transient absorption signal vs. incident power density at $100 \mathrm{ps}$ (black,) $1 \mathrm{~ns}$ (red), and $4 \mathrm{~ns}$ (blue). The MAFA/rub absorbance is extracted at $514 \mathrm{~nm}(c)$ and $553 \mathrm{~nm}(d)$. The dashed lines are added to guide the eye. (e) and (f) Double difference spectra values representing the rubrene triplet state $(514 \mathrm{~nm})$ and polaron state $(553 \mathrm{~nm})$ obtained by subtracting the normalized transient absorbance at $514 \mathrm{~nm}(e)$ and $553 \mathrm{~nm}(f)$ at a delay time of $100 \mathrm{ps}$ from the absorbance at $4 \mathrm{~ns}$. A linear fit at powers $>1 \mathrm{~mW} / \mathrm{cm}^{2}$ is also shown (black) with a slope $\alpha$. 


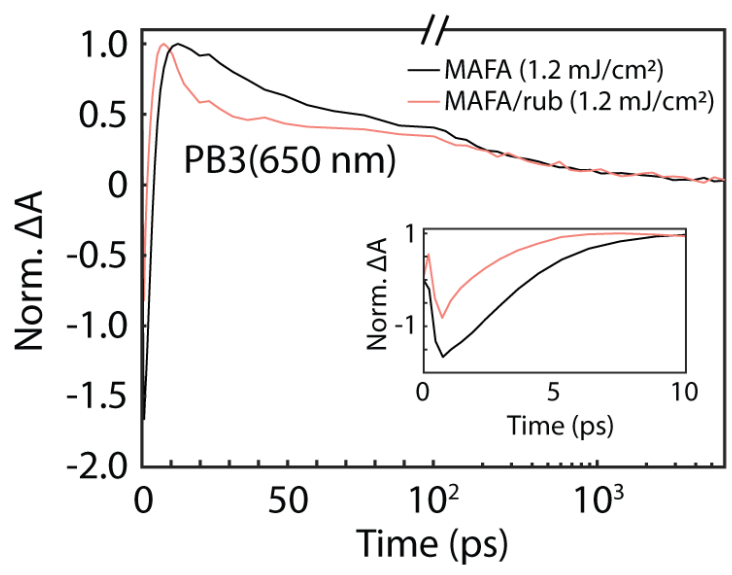

Figure S5: Normalized TA kinetics of MAFA (black) and MAFA/rub (pink) under $700 \mathrm{~nm}$ excitation at a power density of $1.2 \mathrm{~mJ} / \mathrm{cm}^{2}$ extracted at $650 \mathrm{~nm}$. In this spectral region PIA1 and PB3 overlap. The inset is an enlargement of the first 10 ps to highlight the overlapping PB3 dynamics.
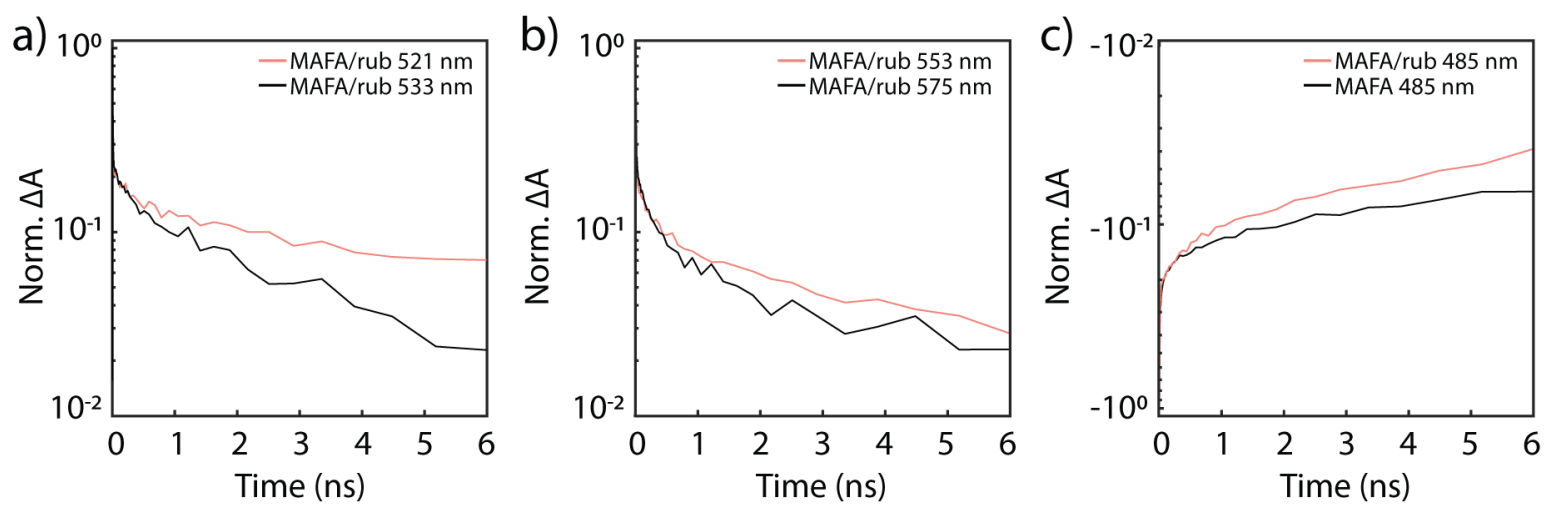

Figure S6: Kinetic traces of MAFA/rub (a,b) and MAFA/rub and MAFA (c) normalized between 80-100 ps to obtain the residual polaron and triplet signal from rubrene. The dynamics in (a) were extracted from the MAFA/rub TA surface (700 nm excitation, $1.2 \mathrm{~mJ} / \mathrm{cm}^{2}$ ) at wavelengths of $521 \mathrm{~nm}$ (pink) and $533 \mathrm{~nm}$ (black). The dynamics in (b) were extracted from the MAFA/rub TA surface at wavelengths of $553 \mathrm{~nm}$ (pink) and $575 \mathrm{~nm}$ (black). The dynamics in (c) were extracted at $485 \mathrm{~nm}$ in the MAFA (black) and MAFA/rub (pink) TA surface. Subtraction of the respective traces and subsequent normalization yield the triplet-related kinetics shown in Figure 3 (for details see above). 


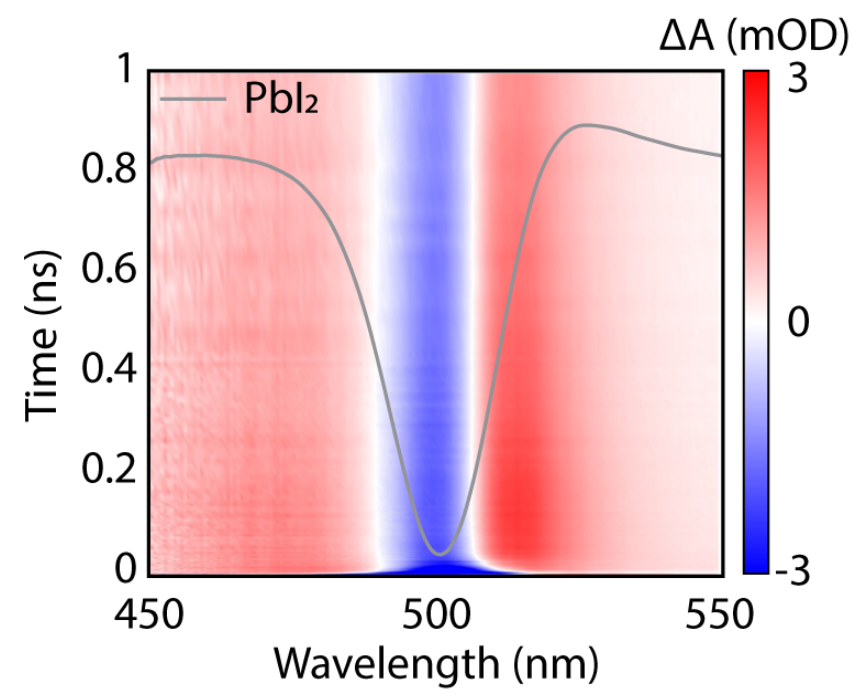

Figure S7: TA surface of a $\mathrm{PbI}_{2}$ film under $420 \mathrm{~nm}$ pump. The PbI 2 TA spectrum at a delay time of 1 ps (gray) is shown inside the TA surface.

\section{SI References}

(1) Wieghold, S.; Bieber, A. S.; VanOrman, Z. A.; Daley, L.; Leger, M.; Correa-Baena, J.-P.; Nienhaus, L. Triplet Sensitization by Lead Halide Perovskite Thin Films for Efficient SolidState Photon Upconversion at Subsolar Fluxes. Matter 2019, 1, 705-719.

(2) Wieghold, S.; Bieber, A. S.; VanOrman, Z. A.; Nienhaus, L. Influence of Triplet Diffusion on Lead Halide Perovskite-Sensitized Solid-State Upconversion. J. Phys. Chem. Lett. 2019, 10, 3806-3811. 